\title{
Improving Nutritional Care in Hospitals Project: (INCHP) establishing a network of nutrition champions
}

\author{
A. W. McKinlay on behalf of The Improving Nutritional Care in Hospitals Project \\ GI Unit, Aberdeen Royal Infirmary, Aberdeen AB25 2ZN
}

In 2001, the Chief Nursing Officer for Scotland instructed NHS Quality Improvement Scotland (NHS QIS) to develop National standards for nutritional care in Scottish hospitals, published in 2003. NHS QIS carried out peer review visits to all Scottish Health Boards in 2005, which showed that all had started to implement the standards but the progress was slow. The problem was how to enforce the standards across the NHS in Scotland, but retain the cooperation and enthusiasm of staff, particularly nurses. In 2007, the Chief Nursing Officer, instigated the INCHP. Membership included public representatives, NHS QIS, NHS Education Scotland, hospital caterers, senior dietitians, senior nurses and representatives of the Royal College of Nurses, and a doctor with an interest in nutrition.

Actions: The INCHP introduced a nutritional toolkit for all nurses emphasising the importance of nutritional screening.

Published a national nutritional specification defining the nutritional content of hospital meals and giving detailed advice to caterers.

Developed a national database of hospital meals with automated nutritional analysis.

Commissioned a web based learning resource for all NHS Scotland employees, and an educational resource for junior doctors.

Initiated a compulsory catering and nutritional care, self-assessment, conducted twice yearly by all health boards.

Introduced a network of Nutrition Champions across all the Health Boards, based on a similar model used in hospital acquired infection, drawn mainly from nursing staff but also dietitians. The champions were brought together from across the country, on a regular basis to form a spontaneous, mutually supportive network which allowed good practice to be shared. The result was a refocusing on the standards across the service.

Results: The results of the NHSQIS national overview, published in April 2010 showed that all boards have made progress and that results were more uniform than in 2006. We believe that the network of Nutrition Champions significantly contributed to this by spreading good practice across regions. NHSQIS found that: MUST is now implemented as a national screening tool for undernutrition across NHS Scotland. A culture of awareness of nutritional care issues is developing across NHS Scotland. A majority of NHS boards have developed and implemented comprehensive policy and strategy documents which address all the requirements of the NHS QIS standards. NHS boards have now implemented an effective system for screening the delivery of food and fluid to patients ${ }^{(1)}$.

Conclusions: Nutrition Champions combine local knowledge and credibility with a national perspective and have greatly enhanced the implementation of the standards. Improvement has been validated by detailed peer review visits ${ }^{(1)}$. We believe that the INCHP represents a possible model that other Health services could use to combat malnutrition in hospitals.

1. NHS QIS National Overview: Food Fluid and Nutritional Care, 2010 http://www.nhshealthquality.org/nhsqis/files/FFNCH_NOV_APR10.pdf 